\title{
Automatic Segmentation of Hepatic Tumors in CT Images using Adaptive EM and Diagnostics of IR Spectroscopy
}

\author{
by K.Lekshmi* and Dr.K.Rubasoundar**
}

\author{
*SSN College of Engineering, Chennai, India, lekshmik@ssn.edu.in \\ ${ }^{* * P . S . R . ~ E n g i n e e r i n g ~ C o l l e g e, ~ S i v a k a s i, ~ I n d i a, ~ r u b a s o u n d a r @ y a h o o . c o m ~}$
}

\section{Abstract (Arial, 9pt, bold)}

The paper introduces the automated segmentation of hepatic tumor from abdominal Computed Tomography images and diagnose it using IR spectroscopy. In this context, a segmentation algorithm for liver tumor is developed, which helps to diagnose the tumor by segmenting the affected tumor area. A hybrid diagnosis method, Adaptive EM is proposed to detect the hepatic lesions automatically by utilizing sequential images of abdominal CT. An area function adaptation scheme that uses EM model grows the clusters without the need for initial selection of clusters. Infrared (IR) spectroscopic imaging of human liver tissue slices has been used to identify and characterize liver tumors, by giving a discriminative model of nucleus and cytoplast of tumor, incorporating texture, layout, and context information efficiently. Both training and testing dataset are preprocessed to produce texton map. The feature responses of each image in the training data set is calculated and are trained efficiently in AdaBoost classifier using Conditional Random Field and Piecewise Training methods. The proposed method provides high classification and segmentation accuracy for nucleus of tumor that are exceedingly textured in IR spectroscopic image. This work also suggests utility for these methods beyond the identification of liver tumors.

Keywords: Adaptive EM, Hepatic lesion, liver tumors, Computed Tomography, AdaBoost, optimized imaging systems, CRF, Piecewise Training method

\section{Introduction}

The paper investigates the problem of achieving automatic detection, recognition, and segmentation of nucleus of tumor in IR spectroscopic images. Specifically, given an image, the system should automatically partition it into semantically meaningful regions each labeled with a specific object class colors as illustrated in Figure 1.
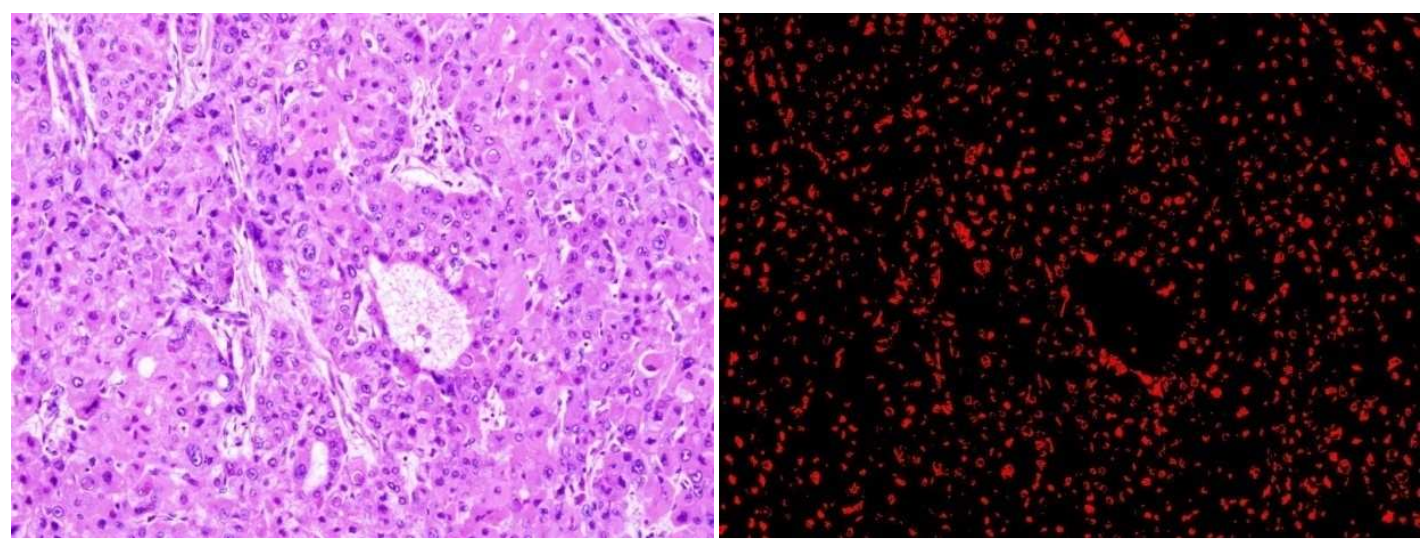

Fig. 1 Example results of new simultaneous Tumor nucleus recognition and segmentation algorithm

The liver is the biggest organ in the human body and today, liver cancer has turned into an exceptionally normal form of cancer with the number of liver malignancy patients growing exponentially. On the off chance that treated in the early stage, liver tumor can be effectively cured. Liver tumor is of two types in particular the primary liver cancer where it begins from the liver and secondary (metastatic) liver cancer which began in an alternate part of the body, for example, pancreas, bowel, stomach, lung or breast and has spread to the liver. Latterly, radiologists physically segment the tumors from computed tomography (CT) scans, and then ascertain the volumes of every slice. Liver tissue, containing a liver metastasis of breast origin (mucinous carcinoma) was surgically removed from a consenting patient and frozen without formalin fixation or dehydration procedures, so that lipids and water remain in the tissues. However, this manual segmentation is a tiresome and prolonged task. Generally, automated segmentation techniques have become popular because of less consumption of time, availability and lack of human data. The tumors, which cause in liver, can result in major health issue. Such tumors can be of benign or malignant type. Noncancerous tumor masses which develop inside liver region are called benign or non-malignant tumors which are generally not life threatening. Where-as cancerous tumor masses, which develop inside liver region are known to be life-threatening. Diagnosis and treatment needs to 
detect these malignant or cancerous tumors precisely. If diagnosed in the early stage of disease, it serves to treat and spare patient from deadly outcomes. The proposed technique automatically segments the liver tumors and its infrared (IR) spectroscopic imaging of human liver tissue slices has been used to identify and characterize liver tumors.

A hybrid diagnosis method, Adaptive EM is proposed to detect the hepatic lesions automatically by utilizing sequential images of abdominal CT. An area function adaptation scheme that uses EM model grows the clusters without the need for initial selection of clusters. As such, the technique can identify $\mathrm{K}$ clusters in an input data set by merging the existing clusters and by the creating new ones while keeping the number of clusters constant. Hepatic lesions are then segmented automatically with necessary morphological operations.

Expectation Maximization (EM) algorithm [9] can be an iterative way for finding maximum likelihood or maximum a posteriori (MAP) estimates of parameters in statistical models, where the model depends on unobserved latent variables. The first step of EM method is performing an expectation (E) step, which creates a function for the expectation of the loglikelihood evaluated using the current estimate for the parameters. The second step is the maximization (M) step, which computes parameters maximizing the expected log-likelihood found on the E step. As these two steps have to be performed alternatively, within one iteration of EM algorithm, first, the probability that a data point comes from a cluster is computed for each data point and each cluster; then its parameters are updated based on the probabilities from the previous step for the distribution of each cluster.

However, with existing imaging modalities such as $x$-rays (plain film, fluoroscopy, and computed tomography $[\mathrm{CT}]$ ), magnetic resonance imaging (MRI), ultrasound (US), single-photon emission computed tomography (SPECT) and positron emission tomography (PET), the unmet clinical need in surgery stems from the fact that visible light cannot penetrate into blood and tissue more than a few hundred microns, due to high photon attenuation from absorbance and scatter. Thus, when a surgeon looks at the surgical field, he/she only sees surface features. For example, with modern surgical techniques, 20 to $25 \%$ of breast cancers are still resected incompletely[2,3] and local recurrence remains unacceptably high at $12-28 \%$. Being able to see structures that need to be resected, such as malignant cells, and structures that need to be avoided, such as blood vessels and nerves, is a profound unmet clinical need.

Near-infrared optical imaging has long been suggested to be a potentially desirable tool for cell and tissue imaging due to the ability to obtain images in which every pixel has a wealth of biochemical information and hence used to detect fluorescently labelled structures during surgery. This type of imaging has the potential to modernize human surgery by providing real-time image guidance to surgeons for tissue that needs to be resected, such as tumors, and tissue that needs to be avoided, such as blood vessels and nerves. Thus it is able to reduce the frequency of positive margins and therefore, the number of second surgeries, by marking or highlighting cancerous tissue for the surgeon. Nevertheless, optimized imaging systems are required for clinical reality.

The characterization of differentially expressed genes between cancerous and normal tissues is an important step in the understanding of tumor genesis. The heterogeneity and instability of human tumors hamper straightforward identification of cancer-causing mutations. Infrared (IR) spectroscopic imaging of human liver tissue slices can be used to identify and characterize liver tumors. Liver tissue is frozen without formalin fixation or dehydration procedures, so that lipids and water remain in the tissues. A set of IR metrics(ratios of various IR peaks) was determined for tumors in fixation-free liver tissues. K-means cluster analysis was used to tell tumor from non-tumor. The aim of the current work is to identify the most important and characteristic IR spectral features associated with tumor-bearing tissues within the liver, with the ultimate goal to develop a method for real-time differentiation of specific cell types associated with diseased liver tissue and normal liver tissue that can be used to assist in the surgical resection of liver tumors.

The challenge is to model the visual variability of a large number of unstructured Tumor nucleus, to be invariant to viewpoint and illumination, and to be robust to occlusion. The focus here is, not only improving the accuracy of segmentation and recognition, but also the efficiency of analyses of the performance of AdaBoost algorithm. The main contributions in this paper are threefold. The texture-layout filter feature is the most significant one. These features record patterns of textons, and exploit the textural appearance of nucleus, its layout, and its textural context. Next a discriminative model is obtained that combines texture-layout filters with lower-level image features, in order to provide a near pixel perfect segmentation of the image. Finally, we demonstrate how to train this model efficiently by exploiting both boosting and piecewise training methods.

The paper is organized as follows. Immediately below, we discuss related work. In Section 2, we describe the image databases used in our experiments. Section 3 introduces the high-level discriminative model, a conditional random field (CRF). Section 4 discusses about system design which consists of texture-layout filters and their combination in a boosted classifier. Finally evaluate and compare with related work and performance of AdaBoost algorithm is discussed in Section 5. Section 6 suggests some novel applications of semantic segmentation and conclusion and future works are provided in Section 7.

\section{Literature Survey}

\subsection{Recording IR spectroscopic imaging data}

The process of recording IR spectroscopic imaging data was stated in [4]. The liver tissue was pretreated by snap freezing in liquid nitrogen, which allowed rapid lowering of the sample to a temperature below $-70{ }^{\circ} \mathrm{C}$. Snap freezing of the liver tissue provided specimen integrity for further analysis. Cryostat sections of $\sim 2-3 \mu \mathrm{m}$ thickness were obtained at $-20{ }^{\circ} \mathrm{C}$. They notably still contain lipi $\mathrm{d}$ and water and therefore have less perturbed biomolecules than with 
fixation. IR spectra were recorded with an imaging FTIR microscope (Perkin Elmer Spotlight 300) with a computercontrolled microscope stage and a liquid-nitrogen-cooled linear array of 16 mercury cadmium telluride (MCT) detectors. Each IR window region was $2,200.0 \mu \mathrm{m}$ vertically by $300.0 \mu \mathrm{m}$ horizontally (352 pixels by 48 pixels). The instrument obtained a full IR spectrum at each image pixel $\left(6.25 \mu \mathrm{m}\right.$ by $6.25 \mu \mathrm{m}$ area) in each window $\left(4 \mathrm{~cm}^{-1} \mathrm{resolution}^{2} 2 \mathrm{~cm}^{-1}\right.$ steps in the range of $750-4000 \mathrm{~cm}^{-1}$, and 16 scans per pixel). A MATLAB function from Perkin Elmer called "fsm_load.m" by Ben Peterson was used to download the spectral data for each of the seven windows and to concatenate them into one large three dimensional matrix of spectra transmittances. The resulting imaging area has 352 rows of pixels and 336 columns of pixels yielding 118,272 pixels in the image. Each image pixel is associated with a distinct IR spectrum.

Considering that there are 1626 wavenumber steps in the spectrum at each of 118,272 pixels, there are more than 192 million measured transmittances to evaluate. IR metrics are devised to reduce the information to a more manageable and meaningful set. We have started with the IR metrics of [4] (for prostate cancer on fixed tissues) and considered a set that might be more useful without fixation in the specific case. Matlab routines facilitate the calculation of a value of each metric at each pixel in the image. The result is a three dimensional matrix of IR metrics, b(i,j,m), where $\mathrm{i}, \mathrm{j}$ are indices over the rows and columns respectively of the image, and $\mathrm{m}$ is an index over the metrics. The metrics reduce the original data, a spectrum of 1626 points at each image pixel, to 64 values at each pixel.

The IR metrics are mostly ratios of absorbance at one band ratio to absorbance at another with corrections for baseline effects. Metric values were calculated at each pixel and scaled into a 0 to 255 range for presentation as a blackand-white bitmap image. Images for metrics $L 1$ and $L 2$ are can be considered separately to highlight different regions of tissue. A composite image can also be created. It is compared to an H\&E stain of a subsequent slice. The transition region between tumor and non-tumor exhibits more contrast in the IR composite image than in the H\&E stain. The IR spectrum is detecting protein changes in the critical region of transition between tumor and non-tumor ones that are not as readily evident in the H\&E stain. The IR work (which is unstained) is clearly capable of distinguishing a tumor and appears useful in characterizing bulk molecular changes in the transition from non-tumor to tumor.

K-means cluster analysis can also be employed to distinguish 5 groups based on the six IR metrics of the 1626 IR spectral points at each image pixel have been reduced to six metric values. K-means cluster analysis uses metric scores at each image pixel to calculate a similarity (or a "distance") between a particular image pixel and the average metric scores of the group, i.e. a group centroid. The routine changes membership of image pixels in groups to minimize the sum of "distances" for each group. The output is an assignment of each image pixel to a group with similar metrics. After the K-means analysis is finished, groups can be organized by "distances" between group centroids.

As illustrated in [1], K-means clustering is a non-hierarchical clustering method. A "hard" class membership for each spectrum is obtained, in which class membership of an individual spectrum can be taken only the values of zero or one. Randomly selected initial cluster centers are iteratively updated, and thus obtain the class membership for each spectrum, assuming well-defined boundaries between the clusters. Let Spectra are illustrated as points in p-dimensional space.

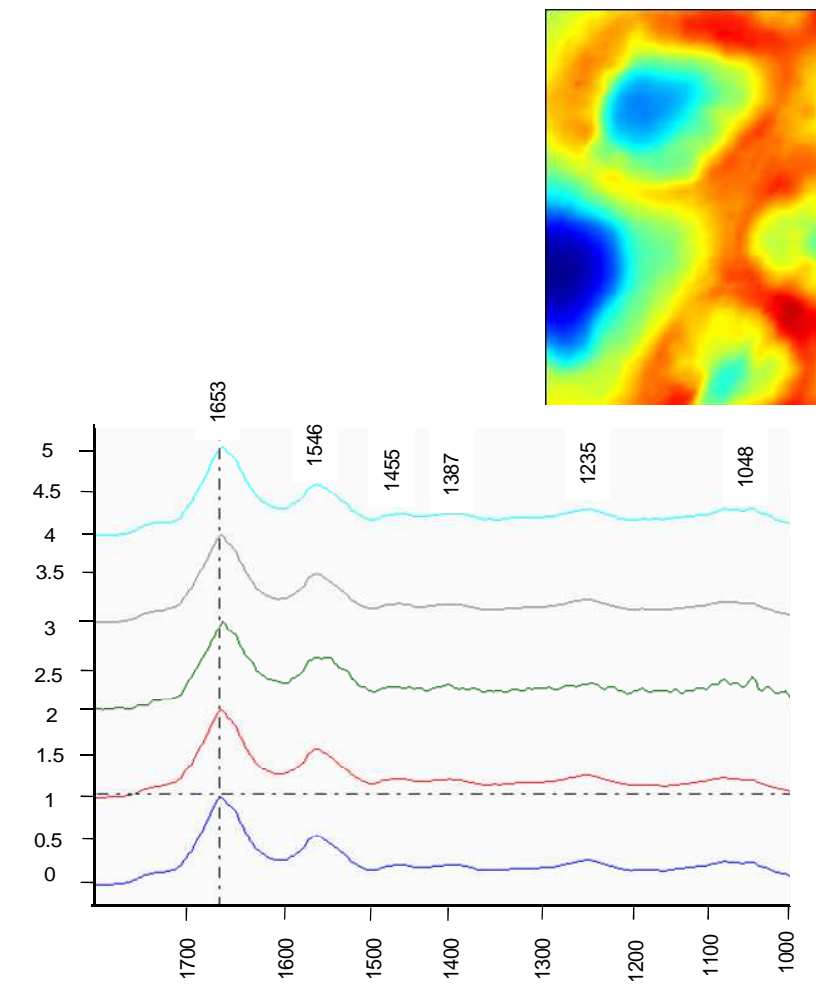


Fig. 2. K-means cluster analysis with 5 groups using the unscaled, top-six metrics(top). The tumor is represented in red groups.

Step 1. Initially choose the number of k points, where each point represents a cluster to be made.

Step 2. Calculate the distance between the points and all objects (spectra).

Step 3. Attribute the closest cluster to each point. recalculated

Step 4. Calculate the centroids of the clusters and distance between centroids and each of the objects are

Step 5. For each object

if the closest centroid is associated with the cluster to which the object currently belongs, then otherwise No change end if

object will switch its cluster membership to the cluster with the closest centroid

End For

Step 6. Repeat the steps 2 to 5 until none of the objects are reassigned

Table 1. IR metric values for 5 groups from K-means Analysis

\begin{tabular}{|l|l|l|}
\hline IR Metric & Non-Tumor & Tumor \\
\hline L1(1744/1548) & 2.06 & 0.882 \\
\hline L2(1744/1244) & 1.68 & 0.483 \\
\hline L3(1742/1256) & 1.34 & 0.312 \\
\hline L4(1080/1548) & 1.38 & 0.17 \\
\hline L5(1080/1244) & 1.21 & 0.344 \\
\hline L6 (1012/1256) & 1.42 & 0.198 \\
\hline
\end{tabular}

\subsection{Various Approaches of Segmentation}

The regions found by bottom-up segmentation were labelled with textual class labels of images, trained in a classifier [6]. Yet, semantic objects are not correlated with such segmentations and hence in proposed method, segmentation and recognition are performed in the same unified framework rather than in two separate steps. At a high computational cost, such a unified approach was presented in [7]. However, using a unary classifier Konishi and Yuille [8] labeled images, spatially coherent segmentations are not achieved.

\section{Image Database}

This implementation should have the ability of obtaining fluorescence images from consent patients. We have obtained 4 normal, 5 hepetocellular carcinoma images, 4 dysplasia images and 4 adenomacarcinoma images from Global Hospital, Chennai. Dr.Mukul, histopathologist and Dr. Balaji of Global Hospital have helped us a lot in getting insight assessment of the extent and number of colorectal liver metastases in fluorescent images.

\section{A Conditional Random Field Model of Classes}

Conditional random field (CRF) model [9] is used to learn the conditional distribution over the class labeling, given an image. To incorporate texture layout, color, location, and edge cues in a single unified model, conditional random field is used. Conditional probability of the class labels $\mathrm{c}$ given an image $\mathrm{x}$ is defined as

$$
\log P(c \mid x, \theta)=\sum_{i} \overbrace{\psi_{i}\left(c_{i}, x_{i} ; \theta_{\psi}\right.}^{\text {textur }})+\pi \overbrace{\pi\left(c_{i}, x_{i} ; \theta_{\pi}\right)}^{\text {color }}+\lambda \overbrace{\left(c_{\mathrm{i}}, \mathrm{i} ; \theta_{\lambda}\right)}^{\text {location }}+\sum_{(\mathrm{i}, \mathrm{j}) \in \varepsilon} \text { edge } \overbrace{\phi\left(\mathrm{c}_{\mathrm{i}}, \mathrm{c}_{\mathrm{j}}, \mathrm{g}_{\mathrm{ij}}(\mathrm{x}) ; \theta_{\phi}\right)}-\log \mathrm{Z}(\theta, \mathrm{x})
$$

where is the set of edges in a 4-connected grid structure, $Z(\theta, x)$ is the partition function which normalizes the distribution, and $\mathrm{i}$ and $\mathrm{j}$ correspond to sites in the graph.

Note that this discriminative model is composed of three unary potentials, depend only on one node $\mathrm{i}$ in the graph, and one pairwise potential depending on pairs of neighbouring nodes in the graph. Therefore the four potential functions and their parameters are defined, before detailing inference and learning. 
http://dx.doi.org/10.21611/qirt.2015.0099

\subsection{Learning the CRF Model}

Three unary potentials and one pairwise potential comprises the four potential functions in this model.

\subsubsection{Texture-Layout Potentials}

The unary texture layout potentials are defined as

$$
\psi\left(\boldsymbol{c}_{\boldsymbol{i}}, X ; \theta_{\psi}\right)=\log \mathrm{P}\left(\boldsymbol{c}_{\boldsymbol{i}} \mid X, i\right)
$$

where $\mathrm{P}\left(\boldsymbol{c}_{\boldsymbol{i}} \mid X, i\right)$ is the normalized distribution given by a boosted classifier. This classifier combines the features of texture-layout filters to model the texture layout, and textural context of the object classes. The texture-layout potentials are proved to be the most powerful term in the CRF, which is shown in the evaluation.

\subsubsection{Color Potentials}

Gaussian Mixture Models (GMMs) are used to represent the color models, where the mixture coefficients depend on the class label. The unary color potentials exploit the color distribution of objects in a particular image. The conditional probability of the color $\mathrm{x}$ of a pixel is given by

$$
P(x \mid c)=\sum_{k} P(x \mid k) P(k \mid c)
$$

with color clusters (mixture components)

$$
P(x \mid k)=\mathcal{N}\left(x \mid \mu_{k}, \sum_{k}\right)
$$

where $\mu_{\mathrm{k}}$ and $\sum_{k}$ are the mean and variance respectively of color cluster $\mathrm{k}$.

\subsubsection{Location Potentials}

This unary location potential performs like a look-up table with an entry for each class and pixel location:

$$
\lambda_{i,}\left(c_{i}, i ; \theta_{\lambda}\right)=\log \boldsymbol{\theta}_{\lambda}\left(c_{i, \hat{\imath}}\right)
$$

where index $\hat{\imath}$ is the normalised version of pixel index $i$, where normalisation lets the images of different sizes: a canonical square on which the image is mapped and $\hat{\imath}$ points to the pixel position within this square. The relatively weak dependence of the class labels on the absolute location of the pixel in the image are confined by these location potentials. Fig.4. illustrates some learned location potentials.

\subsubsection{Edge Potentials}

The edge potentials in the CRF deals severely with neighbouring nodes in the graph having different class labels explicitly, except where there is a corresponding edge in the image. More the darker pixels in image represent more the stronger edge responses and therefore it costs lower. The edge feature $g_{i j}$ measures the difference in color between the neighboring pixels as

$$
g_{i j}=\exp \left(-\beta\left(x_{i}-x_{j}\right)^{2}\right)
$$

where $x_{i}$ and $x_{j}$ are three-dimensional vectors representing the colors of pixels $i$ and $j$ respectively.

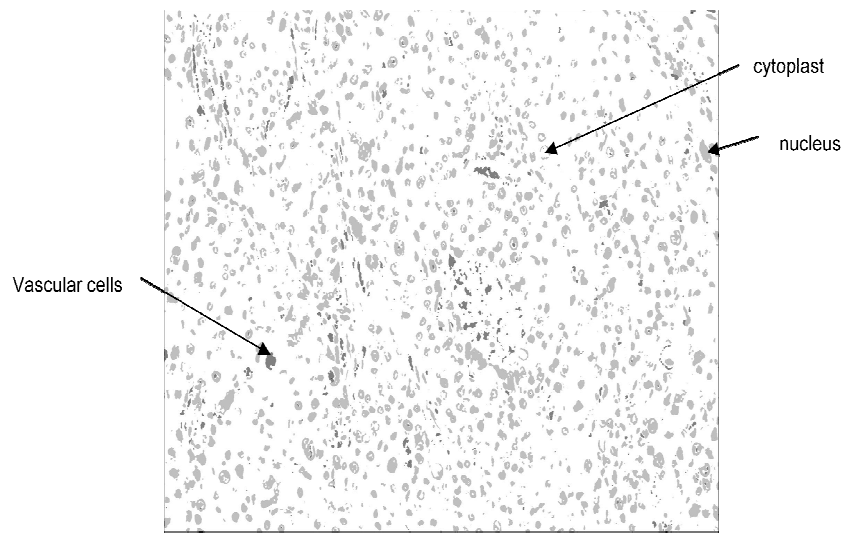


Fig. 3. Example location potentials.

\section{System Design}

The system design describes the models of the proposed system in the following sequence : preprocessing, clustering, feature extraction, building classifier model, performance evaluation.

\subsection{System Architecture}

The overall system architecture is shown below.

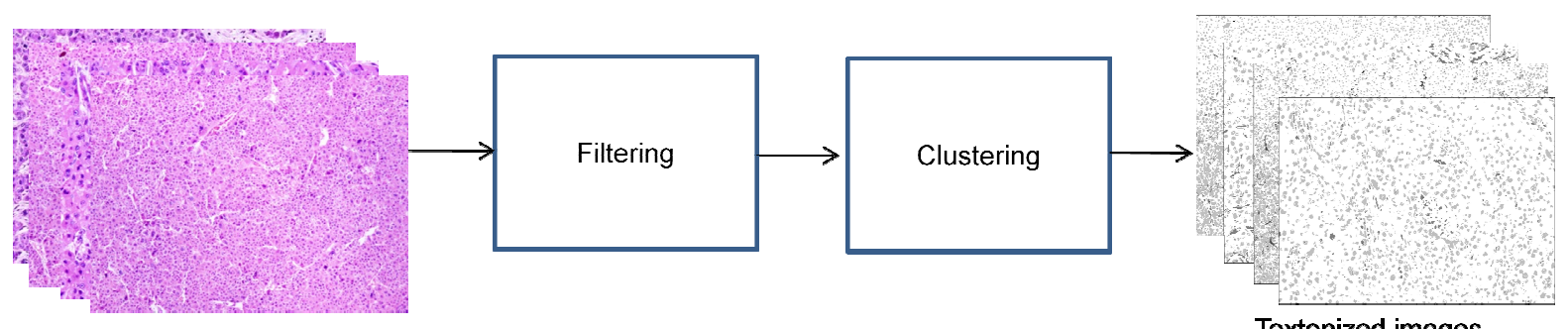

Textonized images

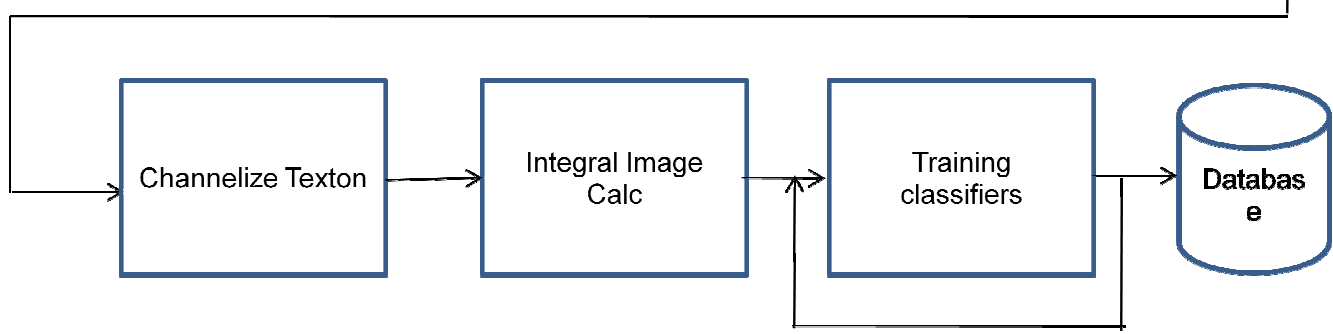

100 Rounds

Fig. 5. Training Phase

\subsection{Textonization}

In this module, training images are given as the input to the 17 dimensional convolution filter bank. The training images are convolved at scale $\mathrm{k}$. The 17D responses for all training pixels are then whitened (to give zero mean and unit covariance), and an unsupervised clustering is performed. Next, we employ the Euclidean-distance K-means clustering algorithm. Finally, each pixel in each image is assigned to the nearest cluster center, producing the texton map.

\subsubsection{Preprocessing}

In preprocessing technique filters are used to reduce the noise. The choice of filter-bank is somewhat arbitrary, as long as it is sufficiently representative. In this project we use 17-D Convolution filter bank. The Gaussians are applied to all three color channels, while the other filters are applied only to the luminance. This filter-bank was determined to have full rank in a singular-value decomposition, and therefore no redundant elements. The filter bank consists of three Gaussian filter, four Laplacian of Gaussian filter (LoG) and four first order derivatives of Gaussians.

\subsubsection{Gaussian Filter}

In case of Gaussian filter shown in Fig. 7 (a), three filters take a sigma value of 1,2,4. All the three Gaussian filter all applied to Hue, Saturation and Value Channel. As a result of this filter we obtain nine filter responses three from each Gaussian Filter. 


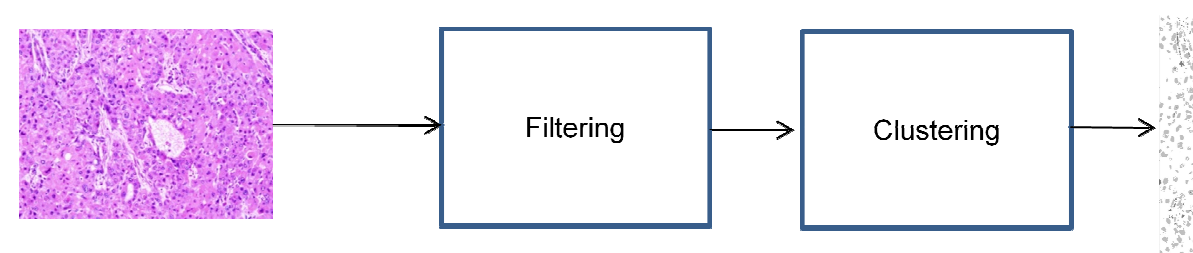

Textonized image

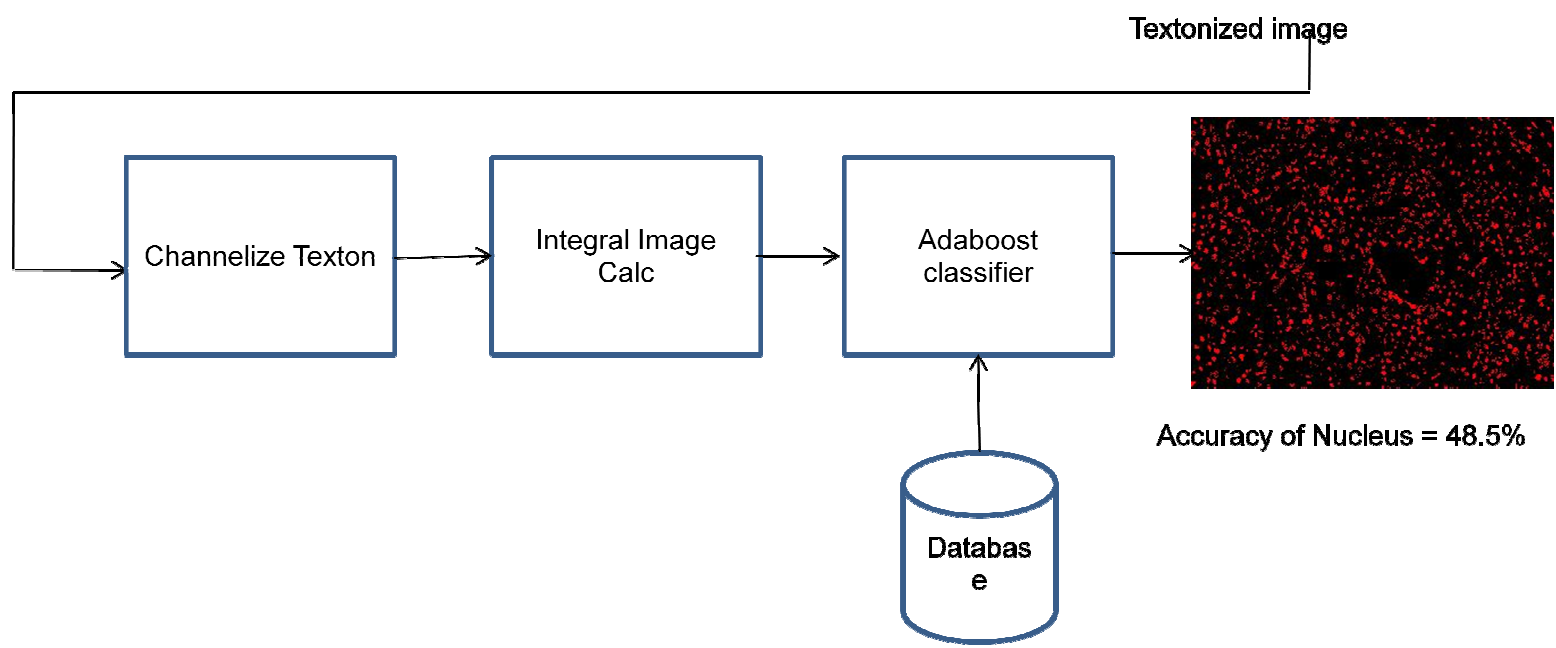

Fig. 6. Testing Phase

\subsubsection{Log Filter}

Four LoG filter with the filter value of 1,2,4,8 correspondingly is used. All the LoG filters are applied to only V Channel. As a result of this filter we get 4 filter response, one response from each LoG filter shown in Fig. 7 (b).

\subsubsection{First Order Derivative Gaussian Filter}

Four filters of first order derivative Gaussian is used ( two x-dimension first order derivative Gaussian filter and two ydimension first order Gaussian derivation) each with two different values of kernels ( sigma =2,4), shown in Fig. 7 (c). All the filters are applied to only $\vee$ Channel. As result it produces four filter responses.

\subsection{Clustering}

K-means clustering aims to partition $\mathrm{n}$ observations into $\mathrm{k}$ clusters in which each observation belongs to the cluster with the nearest mean, serving as a prototype of the cluster. Given a set of observations $(\mathrm{x} 1, \mathrm{x} 2$, , $x \mathrm{n})$, where each observation is a d-dimensional real vector, k-means clustering aims to partition the $n$ observations into $k(n)$ sets $S$ $=\mathrm{S} 1, \mathrm{~S} 2, \ldots, \mathrm{Sk}$ so as to minimize the within-cluster sum of squares (WCSS).

\subsection{Texton Map}

The texton map can be denoted as $\mathrm{T}$ where pixel $\mathrm{i}$ has value $\mathrm{Ti} 1,2, \ldots, \mathrm{K}$. The texton map of an image, containing $\mathrm{K}$ textons, is split into $\mathrm{K}$ channels. An integral image is built for each channel and used to compute texture-layout filter responses in constant time. After running K-means clustering on the filter responses, each pixel in each image is assigned to the nearest cluster center, thus providing the texton map which is shown in system design Fig. 5.

\subsection{Feature Extraction}

In this process, the images in the training dataset are trained and features and nonfeatures are extracted. All those objects which is in database are considered to be features and stored in features model file. Those objects which are not present in object database are considered to be non-features and stored in non-features model file. 


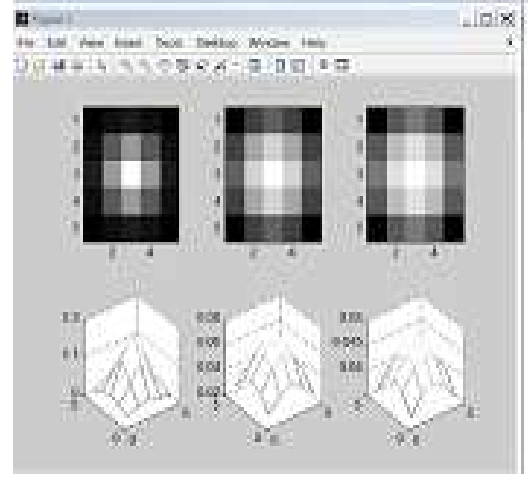

Fig. 7 (a). Gaussian Filter

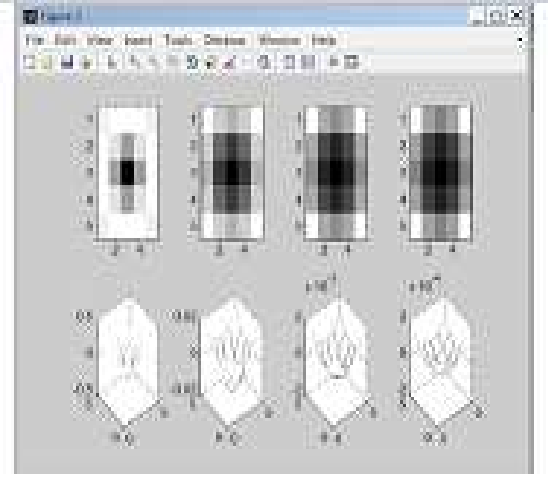

Fig. 7 (b). LOG Filter

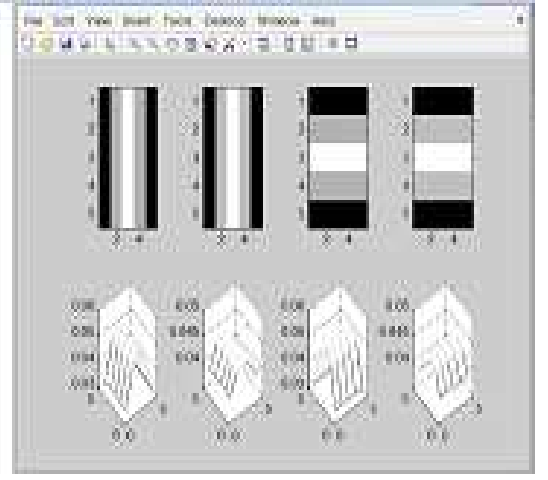

Fig. 7 (c). DOG Filter

\subsection{Subsampling}

The considerable memory and processing requirements make training on a per-pixel basis impractical. Computational expense is reduced by calculating filter responses on a grid (either $3 \times 3$ for the smaller database or $5 \times 5$ for the larger database). The shape filter responses themselves are still calculated at full resolution to enable per-pixel accurate classification at test time. One consequence of this sub-sampling is that a small degree of shift-invariance is learned. On its own, this would lead to inaccurate segmentation at object boundaries. However, when applied in the context of the CRF, the edge and color potentials come into effect to locate the object boundary accurately. As a result of subsampling an image with a source resolution $1600 \times 1200 \times 3$ is been subsampled and reduced to a target resolution of $320 \times 240 \times 3$.

\subsection{AdaBoost Classifier Algorithm}

The boosting process is used for automatic feature selection and learning the texture-layout potentials. Unary classification and feature selection is achieved using shared boosting to give an efficient classifier which can be applied to a large number of classes. A multi-class classifier is learned using an adapted version of the Joint Boosting algorithm. The algorithm iteratively builds a strong classifier as a sum of 'weak classifiers'. Each weak classifier is a decision stump based on a thresholded feature response, and is shared between a set of classes, allowing a single feature to help classify several classes at once. The sharing of features between classes allows for classification with cost sub-linear in the number of classes, and also leads to improved generalization. The learned 'strong' classifier is an additive model summing the classification confidence of $\mathrm{M}$ weak classifiers.

The basic boosting algorithm uses +1 and -1 as output of weak classifiers. The idea of the expansion move algorithm is to reduce the problem of maximizing function with multiple labels to a sequence of binary maximization problems. These subproblems are called alpha-expansions. Suppose that we have a current configuration (set of labels) $\mathrm{C}$ and a fixed label $1,2, \ldots, \mathrm{C}$, where $\mathrm{C}$ is the number of classes. In the alpha-expansion operation, each pixel i makes a binary decision: it can either keep its old label or switch to label. Therefore, we introduce a binary vector $s$ belongs to 0 , 1. Each weak learner is a decision stump based on feature response $v[r, t](i)$ of the form with parameters $(a, b, t h r e s h o l d$, nonfeature index belongs to $C, Q, C, r, t)$. The region $r$ and texton index $t$ together specify the texture-layout filter feature, and $v[r, t](i)$ denotes the corresponding feature response at position $\mathrm{i}$.

For those classes that share this feature (c belongs to $C$ ), the weak learner gives $h_{i}(c)$ belongs to $a+b, \quad b$ depending on the comparison of feature (c belongs to $\mathrm{C}$ ). Round $\mathrm{m}$ chooses a new weak learner by minimizing an error function $\mathrm{J}_{\text {wse }}$ incorporating the weights:

$$
\begin{array}{r}
b=\frac{\sum_{c \in N} \sum_{i} w_{i}^{c} z_{i}^{c}[v(i, r, t) \leq \theta]}{\sum_{c \in N} \sum_{i} w_{i}^{c}[v(i, r, t) \leq \theta]} \\
a+b=\frac{\sum_{c \in N} \sum_{i} w_{i}^{c} z_{i}^{c}[v(i, r, t)>\theta]}{\sum_{c \in N} \sum_{i} w_{i}^{c_{i}^{c}} z_{i}^{c}[v(i, r, t)>\theta]}
\end{array}
$$

\subsection{Testing image}

Here, we input the test image to be processed. If the automatic recognition correctly classifies all the object classes in the image, then we achieve the final output. First the image is textonized to obtain the texton map of the image. From the textonized image we extract the features and non-features in the model file. The features extracted from 
the test set is given as a input to the classifier model which have been generated using AdaBoost algorithm as a result of which we get the semantically segmented image with each object in a image assigned to corresponding color given in the object database.

\section{RESULTS AND COMPARISONS}

In this section we investigate the performance of our system on several challenging datasets, and compare our results with existing work. We first investigate the effect of different aspects of the model, and then give the full quantitative and qualitative results.

\subsection{Boosting Accuracy}

Boosting classifier gradually selects new texture-layout filters to improve classification accuracy. Initially, after 30 rounds of boosting (i.e. 30 texture-layout filters), a very poor classification is given, with low confidence. As more texture layout filters are added, the classification accuracy improves greatly, and after 100 rounds a very accurate classification is given. Note that this illustrates only the texture-layout potentials, and not the full CRF model. As expected, the training error $J_{\text {wse }}(10)$ decreases non-linearly as the number of weak learners increases. Furthermore, Table 2. shows the accuracy of classification with respect to the validation set, which after about 100 rounds flattens out to a value of approximately $48.5 \%$ for tumor nucleus. The accuracy against the validation set is measured as the pixelwise segmentation accuracy, in other words the percentage of pixels that are assigned the correct class label.

Table 2. Performance Analysis of Adaboost classifer in HCC

\begin{tabular}{|l|l|l|}
\hline Classes & Structure/Tissue & Percentage \\
\hline Red & HCC & 48.615 \\
\hline Black & Not identified & 51.385 \\
\hline
\end{tabular}

Table 3. Performance Analysis of Adaboost classifer in Dysplasia

\begin{tabular}{|l|l|l|}
\hline Classes & Structure/Tissue & Percentage \\
\hline Red & Dysplasia & 53.5 \\
\hline Black & Not identified & 46.5 \\
\hline
\end{tabular}

Table 3. Performance Analysis of Adaboost classifer in AdenomaCarcinoma

\begin{tabular}{|l|l|l|}
\hline Classes & Structure/Tissue & Percentage \\
\hline Red & AdenomaCarcinoma & 54.85 \\
\hline Black & Not identified & 45.15 \\
\hline
\end{tabular}

\section{REFERENCES}

1. Zhaomin Chen, Ryan Butke, et al., " Infrared Metrics for Fixation-Free Liver Tumor Detection". NIH Public Access. 2013. [doi:10.1021/jp4073087]

2. Bani MR, Lux MP, Heusinger K, et al., "Factors correlating with reexcision after breast-conserving therapy". Eur J Surg Oncol. 2008

3. Schiller DE, Le LW, Cho BC, et al., "Factors associated with negative margins of lumpectomy specimen: potential use in selecting patients for intraoperative radiotherapy". Ann Surg Oncol. 2008; 15:833-42. [PubMed: 18163174]

4. Fernandez DC, Bhargava R, Hewitt SM, Levin IW., " Infrared spectroscopic imaging for histopathologic recognition". Nat Biotech. 2005; 23:469.

5. P. Duygulu, K. Barnard, N. de Freitas, and D. Forsyth., "Object recognition as machine translation: Learning a lexicon for a fixed image vocabulary". in A. Heyden, G. Sparr, and P. Johansen, editors, Proc. European Conf. on Computer Vision, volume LNCS 2353, pages 97-112. Springer, May 2002.

6. P. Duygulu, K. Barnard, N. de Freitas, and D. Forsyth., " Object recognition as machine translation: Learning a lexicon for a fixed image vocabulary". in A. Heyden, G. Sparr, and P. Johansen, editors, Proc. European Conf. on Computer Vision, volume LNCS 2353, pages 97-112. Springer, May 2002.

7. Z. Tu, X. Chen, A.L. Yuille, and S.C. Zhu., " Image parsing: unifying segmentation, detection, and recognition". In Proc. Int. Conf. on Computer Vision, volume 1, pages 18-25, Nice, France, October 2003.

8. S. Konishi and A. L. Yuille., "Statistical cues for domain specific image segmentation with performance analysis". In Proc. IEEE Conf. Computer Vision and Pattern Recognition, volume 1, pages 125-132, June 2000.

9. J. Lafferty, A. McCallum, and F. Pereira., "Conditional Random Fields: Probabilistic models for segmenting and labeling sequence data". in Proc. Int. Conf. on Machine Learning, pages 282-289, 2001. 
http://dx.doi.org/10.21611/qirt.2015.0099

10. B.C. Russel, A. Torralba, K.P. Murphy, and W.T. Freeman. LabelMe., "A database and webbased tool for image annotation"., Technical Report 25, MIT AI Lab, September 2005. 